\title{
Aplicação em cobertura de complexo hidrossolúvel na formação de mudas de maracujazeiro-azedo
}

\author{
Side dressing water-soluble complex application on yellow passion fruit \\ seedlings formation
}

\section{Mauro Brasil Dias TOFANELLI'; Jair Fernando KOGERATSKI²; Rodrigo Teles dos SANTOS ${ }^{3}$; Jéssica Domingues SCHAFHAUSER ${ }^{4}$}

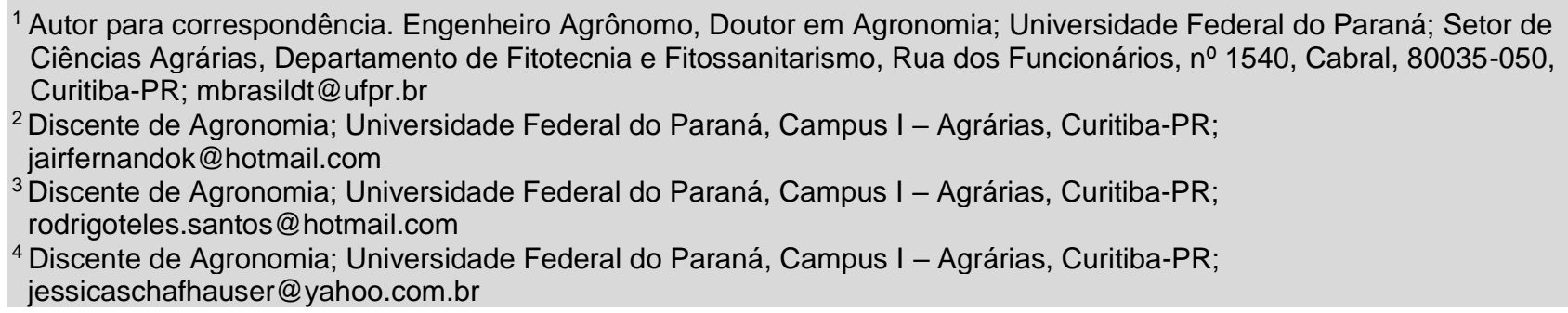

Recebido em: 01-09-2014; Aceito em: 03-11-2015

\begin{abstract}
Resumo
Mudas vigorosas e bem formadas são imprescindíveis para o sucesso da fruticultura. O maracujazeiro-azedo é uma das principais frutíferas tropicais cultivadas no Brasil e é propagado convencionalmente por sementes, porém nem sempre suas mudas atingem desenvolvimento ideal no momento da implantação da cultura. O uso de um produto alternativo como fonte de nutrientes minerais para potencializar 0 crescimento e 0 desenvolvimento das mudas favoreceria sua formação e, consequentemente, seu sistema de produção. Este trabalho teve como objetivo avaliar o crescimento e o desenvolvimento de mudas de maracujazeiro-azedo submetidas à aplicação de fonte de nutrientes minerais alternativa. Para isso, mudas de maracujazeiro-azedo foram formadas em tubetes e acondicionadas em estufa plástica entre os meses de julho e outubro de 2012 . 0 complexo hidrossolúvel $(\mathrm{CH})$ foi aplicado em cobertura mediante irrigação quinzenal com soluções em suas respectivas concentrações de 0; 500; 1.000 e $2.000 \mathrm{mg} \mathrm{L}^{-1}$ sobre as mudas, na presença ou na ausência de adubação química. Aos 80 dias após a semeadura, foram efetuadas as avaliações das características altura, diâmetro do colo, número de folhas, comprimento de raiz e massas das matérias fresca e seca da parte aérea e do sistema radicular. $\mathrm{O} \mathrm{CH}$ favoreceu a qualidade das mudas de maracujazeiro-azedo, especialmente na promoção de melhores resultados em altura e massas das matérias fresca e seca da parte aérea, na concentração de $2.000 \mathrm{mg} \mathrm{L}^{-1}$, porém a adubação química foi importante para sua formação.
\end{abstract}

Palavras-chave adicionais: crescimento; fertilização; maracujá; Passiflora edulis; propagação.

\begin{abstract}
Vigorous and well formatted seedlings are very important for success of the fruit crops. The yellow passion fruit is one of the most important tropical fruit tree cultivated in Brazil whose the propagation usually has occurred by seed, however its seedlings are not always developed sufficiently at the time of planting. Thus, the use of an alternative product as source of mineral nutrients to potentiate seedlings growth and development could be positive for its production system. The objective of this work was to evaluate the growth and development of yellow passion fruit seedlings on water-soluble complex application. For that, the yellow passion fruit seedlings are formatted in plastic tubes into the greenhouse covered with plastic during July to October, 2012. The watersoluble complex (WSC) was applied biweekly in side dressing by solutions on concentrations of $0,500,1000 \mathrm{e}$ $2000 \mathrm{mg} \mathrm{L}^{-1}$ under the seedlings cultivated with or without chemistry fertilization. After 80 days, the characters were evaluated measuring seedling height, stem base diameter, number of leaves, longest root, fresh and dry matter of aerial parts, and fresh and dry matter of roots. The WSC promoted quality of the yellow passion fruit seedlings, especially increasing its height, fresh and dry matter of aerial parts by concentration of $2,000 \mathrm{mg} \mathrm{L}^{-1}$, however chemistry fertilization was important for its formation.
\end{abstract}

Additional keywords: fertilization; growth; Passiflora edulis; propagation; yellow passion fruit. 


\section{Introdução}

O maracujazeiro-azedo ou maracujazeiroamarelo (Passiflora edulis Sims) é uma planta frutífera importante para a fruticultura brasileira, já que o maracujá foi a $11^{\text {a }}$ fruta mais produzida no Brasil em 2012, representando um volume total de 776.097 toneladas (IBGE, 2013). Os pomares de maracujazeiro no Brasil são predominantemente formados por plantas propagadas sexuadamente, e o desenvolvimento de técnicas que visem a melhorar a qualidade das mudas poderia favorecer a implantação da cultura, pois mudas de qualidade são um dos alicerces da fruticultura (Wagner Júnior et al., 2011).

Um dos grandes desafios da agricultura moderna é manter ou até melhorar a qualidade e/ou a quantidade da produção de alimentos, diminuindo custos e adotando medidas ecologicamente corretas; todavia, há falta de tecnologias e de princípios norteadores para os seus respectivos tipos de cultivo (Assis, 2002, Tofanelli \& Silva, 2011). Neste contexto, têm-se buscado novas práticas, técnicas e/ou produtos que sejam tão eficientes quanto os tradicionais ou químicos (sintéticos), mas que, concomitantemente, sejam menos e/ou não degradantes e poluentes.

A fertilização e a nutrição de mudas desempenham relação direta com sua qualidade e é, principalmente, no substrato que se encontram os elementos químicos capazes de prover o crescimento e o desenvolvimento das mudas (Cruz et al., 2008, Silva et al., 2010, Hartmann et al., 2010). Porém, mesmo que o substrato contenha características que o classifiquem como um excelente material, especialmente quanto a suas propriedades químicas, as plantas (mudas) nem sempre apresentam aproveitamento máximo dos elementos químicos nele presentes por influência da interação entre fatores endógenos e exógenos, como a temperatura, umidade, radiação, oxigênio, os balanços nutricionais, energéticos e hormonais, as constituições líquida e estruturais das células, anatomia e morfologia dos órgãos vegetais, idade do vegetal, presença de organismos simbióticos no solo ou substrato, entre outros (Raven et al., 2001, Kirkby \& Römheld, 2007, Taiz \& Zeiger, 2009, Tofanelli \& Silva, 2011).

Práticas de fertilização ou adubação, que consistem no uso de fontes de elementos químicos administrados via solo ou foliar, são adotadas para promover a nutrição das mudas em viveiros, visando a sua boa formação, sendo as fontes minerais sintéticas normalmente as mais utilizadas, porém são onerosas e nem sempre ecologicamente corretas (Assis, 2002, Inocêncio et al., 2009, Sá et al., 2010, Bernardi et al., 2012). Desta forma, vários trabalhos têm sido desenvolvidos para avaliar produtos ou compostos alternativos utilizados como fertilizantes, associados ou não às fontes sintéticas tradicionalmente empregadas na fertilização de mudas de maracujazeiro-azedo (Serrano et al., 2006,
Cruz et al., 2008, Leite et al., 2011, Sousa et al., 2011, Mesquita et al., 2012).

Calcários, fosfatos naturais, rochas moídas, farinhas, vinhotos, xistos, esgotos e outros são algumas alternativas de fornecimento de macro e micronutrientes às plantas em detrimento aos fertilizantes sintéticos (Pereira \& Vitti, 2004, Valadão et al., 2006, Tofanelli \& Silva, 2011, Almeida et al., 2012).

Muitos fertilizantes agrícolas possuem sua formulação baseada em mistura de compostos sintéticos, e dentre os mais comuns, cita-se o adubo NPK, que no Brasil, inclusive, é muito dependente das importações (Profeta \& Braga, 2011). Seguindo uma tendência mundial de substituição destes fertilizantes químicos por adubos cuja fonte não seja sintética, produtos têm aparecido no mercado como alternativa para a agricultura cuja segurança alimentar, ambiental e social ganha destaque. Neste contexto, citam-se alguns produtos fabricados a partir de fontes naturais ou residuais cujo resultado é um complexo de elementos minerais a serem disponibilizados para as plantas em suas diversas formas de aplicação (Almeida et al, 2014, Almeida et al., 2012, Leite et al, 2011, Sousa et al., 2011, Rodolfo Junior et al., 2008).

Um destes produtos é o Systema Clorofila ${ }^{\circledR}$, que é sintetizado a partir de reações básicas formadoras de quelatos de ácido cítrico, carbonatos e bicarbonatos de metais, contendo o Fosfato de Araxá adicionado como lubrificante e o Calcário de Xisto como espessante (Calcário de Xisto Tensoativo + Fosfato Natural Ativado), cujo resultado é a formação de citrato complexo nanômero hidrossolúvel tensoativo com fosfato natural contendo em sua composição: N, P, K, Ca, Mg, S, $\mathrm{Mn}, \mathrm{Fe}, \mathrm{Cu}, \mathrm{B}, \mathrm{Ni}, \mathrm{Se}, \mathrm{Li}, \mathrm{Zn}$, Co, Mo e $\mathrm{Na}$ (Müller-Stecz Indústria e Comércio, 2011). Dentre as vantagens deste composto, destacam-se o fato de sua fabricação não envolver produtos sintéticos e ser este produto de fácil aplicação em virtude de sua alta solubilidade, sendo facilmente dissolvido em solução sem provocar entupimentos e corrosão em implementos agrícolas, mas, por outro lado, as doses recomendadas de aplicação são de baixa concentração, fornecendo macronutrientes em níveis provavelmente insuficientes às plantas.

Como há, às vezes, incerteza sobre o verdadeiro efeito destes fertilizantes alternativos sobre as culturas e sua eficácia em relação aos já consagrados adubos sintéticos. É preciso então testar tais produtos em todos os sistemas de cultivo e, no caso da fruticultura, na etapa de formação de mudas, que é fundamental para o sucesso desta atividade. Neste sentido, o presente trabalho teve como objetivo avaliar o efeito da aplicação de concentrações do complexo hidrossolúvel como fonte de nutrientes minerais no crescimento de mudas de maracujazeiro-azedo como insumo alternativo ao fertilizante sintético. 


\section{Material e métodos}

O experimento foi desenvolvido entre os meses de julho e outubro de 2012, no Departamento de Fitotecnia e Fitossanitarismo do Setor de Ciências Agrárias da Universidade Federal do Paraná (Curitiba-PR), localizado nas coordenadas $25^{\circ} 24^{\prime} 38^{\prime \prime}$ de latitude Sul e 49¹4'56" de longitude Oeste, sendo a fase formação das mudas conduzida em estufa tipo capela com coberta de filme plástico de polietileno de baixa densidade, com $150 \mu \mathrm{m}$ de espessura.

As sementes do maracujazeiro do grupo amarelo azedo foram obtidas de frutos selecionados e adquiridos no mercado local. Estas foram extraídas dos frutos e polvilhadas com pó de cal para a retirada da mucilagem que as envolve, sendo, posteriormente, lavadas e colocadas para secar à sombra, por 48 horas. A semeadura foi realizada em tubetes de $120 \mathrm{~cm}^{3}$ de volume, $3,6 \mathrm{~cm}$ de diâmetro e $14 \mathrm{~cm}$ de comprimento, contendo substrato à base de terra de barranco + areia lavada + esterco bovino $(4: 1: 1 \mathrm{v} / \mathrm{v})+1 \mathrm{~kg}$ de calcário dolomítico por $\mathrm{m}^{3} \mathrm{da}$ mistura, colocando-se 2 sementes por tubete, à profundidade de $1 \mathrm{~cm}$, sendo que o substrato não foi incubado após a aplicação do calcário. Os tubetes, então, foram colocados em bandejas plásticas com 96 células. Após a emergência e a estabilização dos "seedlings", que ocorreu quando a maioria das mudas atingiu $5 \mathrm{~cm}$ de altura, sendo realizado 0 desbaste e preservando-se os melhores indivíduos. As mudas foram mantidas na estufa plástica, onde receberam diariamente duas irrigações manuais com auxílio de um regador.

Foi retirada uma amostra do substrato antes de receber o calcário para análise de rotina, cujos resultados foram: $\mathrm{pH}\left(\mathrm{CaCl}_{2}\right): 6,3 ; \mathrm{P}: 232,0 \mathrm{mg} \mathrm{dm}^{-3}$; $\mathrm{K}^{+}: 1,68 \mathrm{cmol}_{\mathrm{c}} \mathrm{dm}^{-3} ; \mathrm{Ca}^{+2}: 11,19 \mathrm{cmol}_{\mathrm{c}} \mathrm{dm}^{-3} ; \mathrm{Mg}^{+2}$ : $3,41 \mathrm{cmol}_{\mathrm{c}} \mathrm{dm}^{-3} ; \mathrm{Al}^{+3}$ : $0,0 \mathrm{cmol}_{\mathrm{c}} \mathrm{dm}^{-3} ; \mathrm{H}^{+}+\mathrm{Al}^{+3}$ : $3,0 \mathrm{cmol}_{\mathrm{c}} \mathrm{dm}^{-3}$; soma de bases (SB): $16,28 \mathrm{cmol}_{\mathrm{c}} \mathrm{dm}^{-3}$; CTC a pH 7,0 (T): 19,28 $\mathrm{cmol}_{\mathrm{c}} \mathrm{dm}^{-3} ; \mathrm{C}: 36,3 \mathrm{~g} \mathrm{dm}^{-3}$, e saturação de base $(\mathrm{V}): 84 \%$.

Os tratamentos experimentais foram constituídos pela aplicação do complexo hidrossolúvel $(\mathrm{CH})$ nas concentrações 0; 500; 1.000 e $2.000 \mathrm{mg} \mathrm{L}^{-1}$, sendo a concentração de $500 \mathrm{mg} \mathrm{L}^{-1}$ equivalente à recomendada pela fabricante, e pela presença ou ausência de adubação química, definindo um esquema fatorial $4 \times 2$. O delineamento experimental foi o inteiramente casualizado, com três repetições, dispostas nas bandejas em parcelas com dezesseis mudas cada uma, distribuídas de maneira que se formasse um quadrado ( 4 mudas $\times 4$ mudas), sendo úteis as quatro centrais.

$\mathrm{O} \mathrm{CH}$ (Systema Clorofila ${ }^{\circledR}$ ) foi fornecido pela fabricante Müller Stecz Indústria (PiraquaraPR) em pastilhas de 5 gramas. Conforme informações do fabricante, tal produto é um potencializador do crescimento vegetal, pois se trata de um complexo hidrossolúvel cuja composição provém de reações básicas formadoras de quelatos de ácido cítrico, carbonatos e bicarbonatos de metais, com o Fosfato de Araxá adicionado como lubrificante e o Calcário de Xisto como espessante (Calcário de Xisto Tensoativo + Fosfato Natural Ativado). A composição marcada (p/v) do complexo é de $\mathrm{Mg}(12 \%)$, $\mathrm{N}(9,5 \%), \mathrm{K}(9 \%)$, Na $(9 \%)$, Ca $(5 \%), \mathrm{S}(4,5 \%), \mathrm{P}$ $(3,5 \%), \mathrm{Mn}(1 \%)$, Fe (1\%), Cu (1\%), B $(0,5 \%), \mathrm{Ni}$ $(0,5 \%)$, Se $(0,05 \%)$, Li $(0,02 \%), \mathrm{Zn}(0,02 \%)$, Co $(0,01 \%)$ e Mo $(0,01 \%)$. O produto, então, é resultado da reunião por síntese dos reagentes com componentes naturais produzidos pela natureza, como o Calcário de Xisto e o Fosfato de Araxá.

Para a aplicação do fertilizante, foram preparadas soluções diluindo-se o produto em água destilada, conforme suas respectivas concentrações e, posteriormente, aplicadas quinzenalmente sobre as mudas mediante irrigação manual com uso de regador. $\mathrm{O}$ volume de aplicação de todas as soluções de $\mathrm{CH}$ foi na base de $20 \mathrm{~mL}^{\text {muda}}{ }^{-1}$.Já 0 tratamento "adubação química convencional" foi realizado mediante adubação de base, conforme Lima (2002) e Silva (2004), adicionando-se ao substrato da muda $5,0 \mathrm{~kg}$ de Superfosfato Simples $\left(20 \%\right.$ de $\left.\mathrm{P}_{2} \mathrm{O}_{5}\right)$ e $0,5 \mathrm{~kg}$ de Cloreto de Potássio $(60 \%$ $\mathrm{KCl})$ por $\mathrm{m}^{3}$ e adubações de cobertura com aplicações quinzenais de $\mathrm{N}$ mediante irrigação com solução de Ureia a 1\% até a coleta dos dados, sendo a primeira adubação nitrogenada realizada logo após a operação de desbaste.

Aos 80 dias após a semeadura (DAS), foram coletados os dados das mudas do maracujazeiroazedo, avaliando-se a altura da muda $(\mathrm{cm})$, o diâmetro do colo $(\mathrm{mm})$, o número de folhas por muda, o comprimento da maior raiz $(\mathrm{cm})$, a massas das matérias fresca e seca $(\mathrm{g})$ da parte aérea e das raízes, calculando-se as médias de cada repetição. Para a determinação da altura da muda, utilizou-se de uma régua graduada em milímetro, medindo a distância entre o colo e o ápice da muda. $\mathrm{O}$ diâmetro do colo foi medido a $1 \mathrm{~cm}$ acima da superfície do substrato, com auxílio de um paquímetro analógico com a leitura dada em milímetro. Para as medições no sistema radicular, procedeu-se à retirada das mudas dos tubetes para posterior destorroamento e lavagem das raízes. A medição do comprimento da maior raiz foi realizada com uma régua graduada em milímetro. A massa da matéria fresca, tanto da parte aérea quanto das raízes, foi determinada separandose estas duas porções mediante corte na região do colo, destorroamento do substrato e lavagem das raízes, e posterior pesagem em balança de precisão. Já a massa da matéria seca foi determinada colocando-se os respectivos materiais dentro de sacos de papel individuais, em estufa a $60{ }^{\circ} \mathrm{C}$, até que se estabilizasse o peso de cada amostra para, então, efetuar sua pesagem na balança de precisão (Sampaio, 1998).

As análises estatísticas foram realizadas mediante análise de variância dos dados observados, 
procedendo-se à comparação de médias pelo teste de Tukey, a $5 \%$ de significância, para os fatores de variação. Quando necessário, foi realizada regressão polinomial para avaliar o efeito das concentrações do produto mediante estimativas obtidas pelas equações, considerando significativos os ajustes com probabilidade $p<0,05$ e coeficiente de determinação $\left(R^{2}\right) \geq 0,80$.

\section{Resultados e discussão}

Houve efeito significativo tanto do complexo hidrossolúvel $(\mathrm{CH})$ quanto da adubação química convencional (AQC) para grande maioria das características avaliadas nas mudas de maracujazeiro-azedo no presente trabalho, porém a interação destes dois fatores não foi significativa em todas elas (Tabela 1).

Tabela 1 - Altura (AL), diâmetro do colo (DI), número de folhas por muda (NF), massa da matéria fresca da parte aérea (MFA), massa da matéria seca da parte aérea (MSA), comprimento de raiz (CR), massa da matéria fresca das raízes (MFR) e massa da matéria seca das raízes (MSR) de mudas de maracujazeiroazedo submetidas à aplicação em cobertura de diferentes concentrações do complexo hidrossolúvel $(\mathrm{CH})$ e de adubação química convencional (AQC). Height of plant (AL), stem base diameter (DI), number of leaves (NF), fresh matter of aerial parts (MFA), dry matter of aerial parts (MSA), longest root (CR), fresh matter of roots (MFR), and dry matter of roots (MSR) evaluated in yellow passion fruit seedlings fertilized with chemistry fertilizer (AQC).

\begin{tabular}{lcccccccc}
\hline \multicolumn{1}{c}{ Fatores } & $\begin{array}{c}\mathrm{AL} \\
(\mathrm{cm})\end{array}$ & $\begin{array}{c}\mathrm{DI} \\
(\mathrm{cm})\end{array}$ & $\begin{array}{c}\mathrm{NF} \\
\left(\mathrm{N} N \text { muda }^{-1}\right)\end{array}$ & $\begin{array}{c}\mathrm{CR} \\
(\mathrm{cm})\end{array}$ & $\begin{array}{c}\mathrm{MFA} \\
(\mathrm{g})\end{array}$ & $\begin{array}{c}\text { MSA } \\
(\mathrm{g})\end{array}$ & $\begin{array}{c}\text { MFR } \\
(\mathrm{g})\end{array}$ & $\begin{array}{c}\text { MSR } \\
(\mathrm{g})\end{array}$ \\
\hline $\mathrm{CH}\left(\mathrm{mg} \mathrm{L}^{-1}\right)$ & & & & & & & & \\
\hline 0 & $7,44 \mathrm{~b}$ & $1,69 \mathrm{c}$ & $7,00 \mathrm{a}$ & $15,73 \mathrm{a}$ & $1,29 \mathrm{a}$ & $0,25 \mathrm{a}$ & $1,51 \mathrm{~b}$ & $0,13 \mathrm{c}$ \\
500 & $8,19 \mathrm{ab}$ & $2,21 \mathrm{a}$ & $6,58 \mathrm{a}$ & $14,32 \mathrm{~b}$ & $1,36 \mathrm{a}$ & $0,28 \mathrm{a}$ & $1,79 \mathrm{a}$ & $0,17 \mathrm{a}$ \\
1.000 & $8,11 \mathrm{ab}$ & $1,86 \mathrm{~b}$ & $7,08 \mathrm{a}$ & $15,84 \mathrm{a}$ & $1,43 \mathrm{a}$ & $0,28 \mathrm{a}$ & $1,54 \mathrm{ab}$ & $0,14 \mathrm{bc}$ \\
2.000 & $9,09 \mathrm{a}$ & $2,32 \mathrm{a}$ & $6,58 \mathrm{a}$ & $14,44 \mathrm{~b}$ & $1,66 \mathrm{a}$ & $0,33 \mathrm{a}$ & $1,63 \mathrm{ab}$ & $0,16 \mathrm{~b}$ \\
\hline $\mathrm{AQC}$ & & & & & & & & \\
\hline Sem & $7,52 \mathrm{a}$ & $1,95 \mathrm{a}$ & $6,38 \mathrm{a}$ & $15,04 \mathrm{a}$ & $1,14 \mathrm{a}$ & $0,26 \mathrm{a}$ & $1,47 \mathrm{a}$ & $0,14 \mathrm{a}$ \\
Com & $8,89 \mathrm{~b}$ & $2,09 \mathrm{~b}$ & $7,25 \mathrm{~b}$ & $15,13 \mathrm{a}$ & $1,73 \mathrm{~b}$ & $0,31 \mathrm{~b}$ & $1,76 \mathrm{~b}$ & $0,16 \mathrm{~b}$ \\
1 Interação & & & & & & & & \\
CH x AQC & 0,5078 & 0,3181 & 0,0794 & 0,2090 & 0,3247 & 0,6975 & 0,8756 & 0,4210 \\
DP & 0,210 & 0,024 & 0,135 & 0,092 & 0,042 & 0,001 & 0,048 & 0,004 \\
\hline
\end{tabular}

${ }^{1}$ Valores de F; Médias seguidas por letras distintas na coluna, diferem entre si pelo teste de Tukey, a 5\% de probabilidade; DP - Desvio-padrão.

Não foi observado efeito do $\mathrm{CH}$ para o número de folhas (dados não apresentados). Contudo, para as características altura, massas das matérias fresca e seca da parte aérea, foi observado efeito das concentrações de $\mathrm{CH}$ (Figuras 1, 2 e 3). Na Tabela 1, observa-se, então, que o diâmetro do colo foi maior quando as mudas de maracujazeiroazedo foram submetidas aos tratamentos com 500 ou $2.000 \mathrm{mg} \mathrm{L}^{-1}$. Para o comprimento das raízes, a aplicação do $\mathrm{CH}$ não promoveu aumento desta característica em relação à testemunha. Já para a massa da matéria fresca das raízes, a concentração de $500 \mathrm{mg} \mathrm{L}^{-1}$ foi a que apresentou resultado superior em relação à testemunha, ao passo que as mudas fertilizadas com $500 \mathrm{mg} \mathrm{L}^{-1} \mathrm{Ou} 2.000 \mathrm{mg} \mathrm{L}^{-1}$ do $\mathrm{CH}$ obtiveram maior massa da matéria seca de raízes quando comparadas às que não receberam o produto.

Os melhores resultados obtidos quando se aplicou o $\mathrm{CH}$ (Tabela 1) podem ser explicados pelo fato de este produto conter em sua formulação teores de macro e micronutrientes, e quando aplicado em cobertura sobre as mudas na maior concentração estes foram suficientes para estimular o maior crescimento das mesmas quando comparadas às que não receberam aplicação com o $\mathrm{CH}$. Serrano et al. (2006) observaram altas extrações de N, K, Ca, $\mathrm{Mn}, \mathrm{Fe}, \mathrm{B}, \mathrm{Zn}$ e $\mathrm{Cu}$ em mudas de maracujazeiroazedo, sendo os três primeiros os mais extraídos, ressaltando-se, então, o fato de estes serem uns dos elementos minerais mais presentes no $\mathrm{CH}$

A altura e as massas das matérias fresca e seca da parte aérea obtiveram regressão polinomial significativa para o efeito das concentrações do $\mathrm{CH}$. $\mathrm{Na}$ Figura 1, observa-se que houve tendência de incremento linear na altura com 0 aumento das concentrações, estimando-se a altura de $9,1 \mathrm{~cm}$ para a concentração de $2.000 \mathrm{mg} \mathrm{L}^{-1}$ do $\mathrm{CH}$, sugerindo inclusive que dosagens mais elevadas poderiam promover crescimento ainda maior das mudas. 


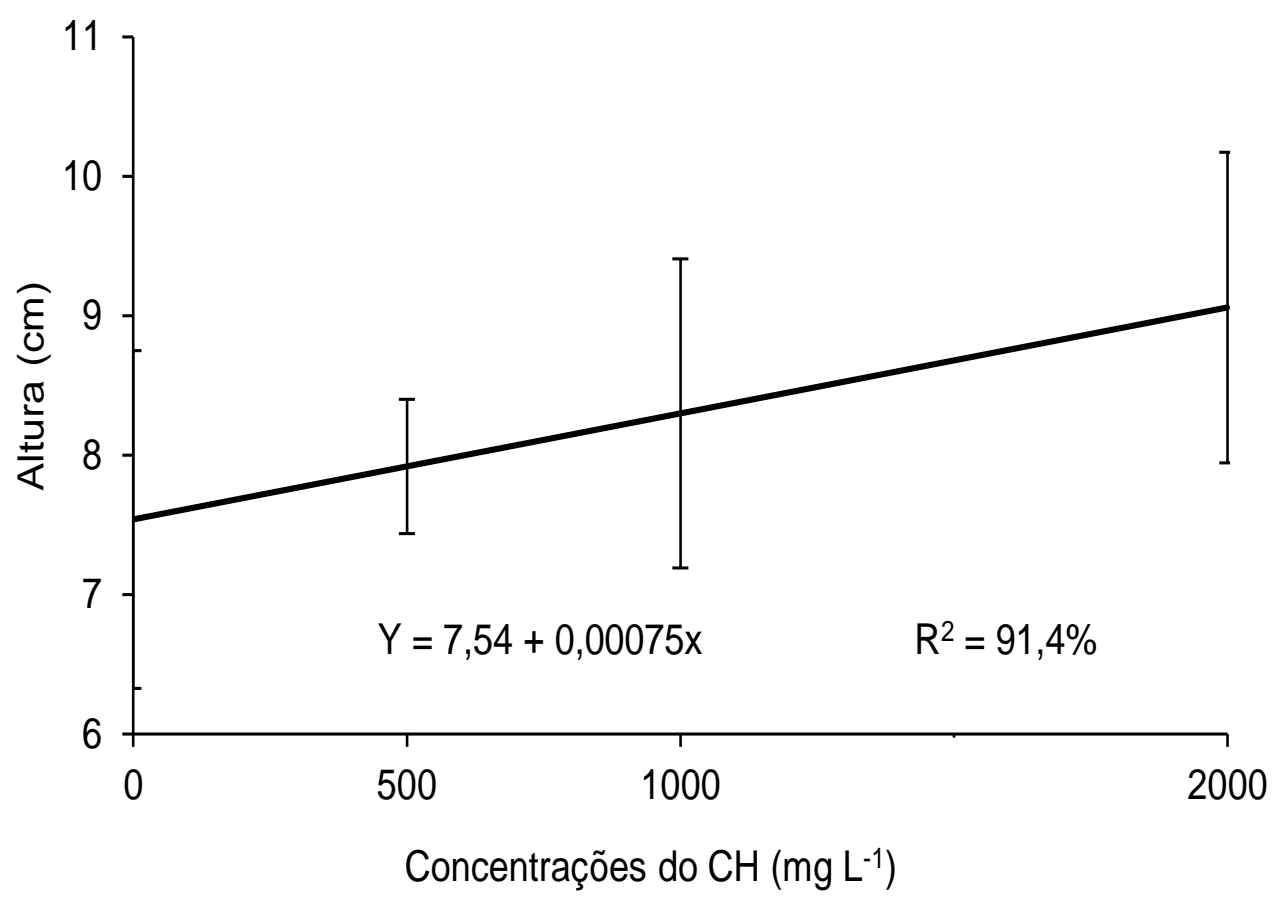

Figura 1 - Altura de mudas de maracujazeiro-azedo submetidas ao tratamento com complexo hidrossolúvel $(\mathrm{CH})$. Médias dos tratamentos com $\mathrm{CH}$ e adubação química. Height of yellow passion fruit seedlings fertilized with water-soluble complex $(\mathrm{CH})$. Averages of treatments with the $\mathrm{CH}$ and the chemistry fertilizer. $(\mathrm{F}=0,0017)$.

$\mathrm{O} \mathrm{N}$ é componente do $\mathrm{CH}$, e seu teor é um dos maiores entre os elementos minerais que constituem o produto, o que pode ter contribuído para estimular o maior crescimento das mudas do maracujazeiro-azedo à medida que se aumentavam as concentrações do produto. Seguindo o mesmo pensamento, outros elementos também têm sido relatados como importantes para o crescimento e a qualidade da muda de maracujazeiro-azedo, citando-se neste caso o K, P, Ca, Mg, S, Fe, Mn e B (Pereira et al., 2000, Almeida et al., 2006, Natale et al., 2006, Serrano et al., 2006, Mendonça et al., 2007, Albuquerque et al., 2010, Sousa et al., 2011, Almeida et al., 2012), estes também presentes na composição do $\mathrm{CH}$.

Costa et al. (2011), adicionando composto orgânico comercial no substrato em diferentes volumes de recipientes para formação de mudas de maracujazeiro-azedo, obtiveram 6,$1 ; 8,6$ e $7,6 \mathrm{~cm}$ de altura em três ambientes de cultivo diferentes: estufa com filme de polietileno e tela termorrefletora de $50 \%$ de sombra, viveiro agrícola de tela de monofilamento com $50 \%$ de sombra e viveiro agrícola de tela termorrefletora com $50 \%$ de sombra, respectivamente. Já Gurgel et al. (2007) estimaram alturas crescentes que variaram de 10,8 até $17,2 \mathrm{~cm}$ em mudas de maracujazeiro-azedo conforme se aumentavam as doses de composto orgânico $(0 \mathrm{a}$ $45 \mathrm{~kg} \mathrm{~m}^{-3}$ ) adicionadas no substrato.

Na característica diâmetro do colo, obtevese com a regressão aumento linear de seu valor à medida que se aumentavam as dosagens do $\mathrm{CH}$, obtendo-se o diâmetro de 2,3 mm na maior dosagem (Figura 2).

As regressões para as características número de folhas e comprimento de raiz das mudas de maracujazeiro-azedo demonstraram queda nos valores estimados à medida que se aumentavam as dosagens do $\mathrm{CH}$ (Figuras 3 e 4).

Já para massa da matéria fresca da parte aérea, observou-se aumento linear conforme se aumentavam as concentrações do $\mathrm{CH}$ (Figura 5), apresentando neste caso tendência similar ao observado para a altura das mudas em relação às dosagens do $\mathrm{CH}$ aplicadas. $\mathrm{Na}$ concentração de $2.000 \mathrm{mg} \mathrm{L}^{-1}$, a massa da matéria fresca da parte aérea foi de $1,64 \mathrm{~g}$.

Considerando que o $\mathrm{N}$ está presente em muitos compostos celulares, que o Ca é componente da lamela média celular e que o Mg está presente na molécula da clorofila (Raven et al., 2001, Lima, 2002, Taiz \& Zeiger, 2009, Tofanelli \& Silva, 2011, Almeida, 2012) e que os teores destes elementos são uns dos mais altos no $\mathrm{CH}$, possivelmente, as soluções mais concentradas deste produto, quando aplicadas nas mudas, deram melhor suporte para a maior produção de massa da matéria vegetal.

Leite et al. (2011) também obtiveram incremento em massa fresca de mudas de maracujazeiroazedo formadas em substrato enriquecido com triturado seco de jitirana (Merremia aegyptia L.) e atribuíram isso ao nitrogênio fornecido por este material adicionado ao substrato. 


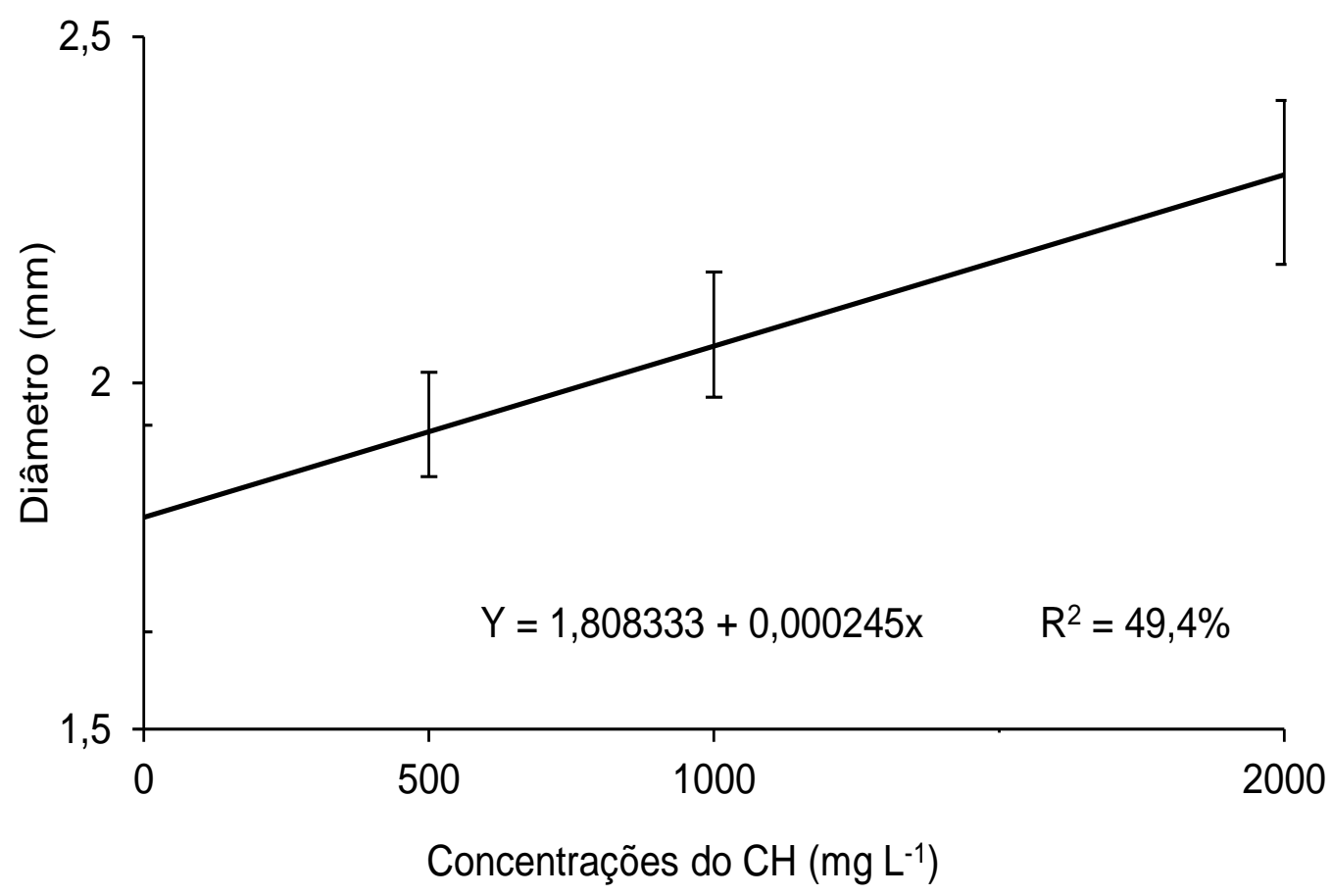

Figura 2 - Diâmetro do colo de mudas de maracujazeiro-azedo submetidas ao tratamento com complexo hidrossolúvel $(\mathrm{CH})$. Médias dos tratamentos com $\mathrm{CH}$ e adubação química. Stem base diameter of yellow passion fruit seedlings fertilized with water-soluble complex (CH). Averages of treatments with the $\mathrm{CH}$ and the chemistry fertilizer. $(F=0,4300)$.

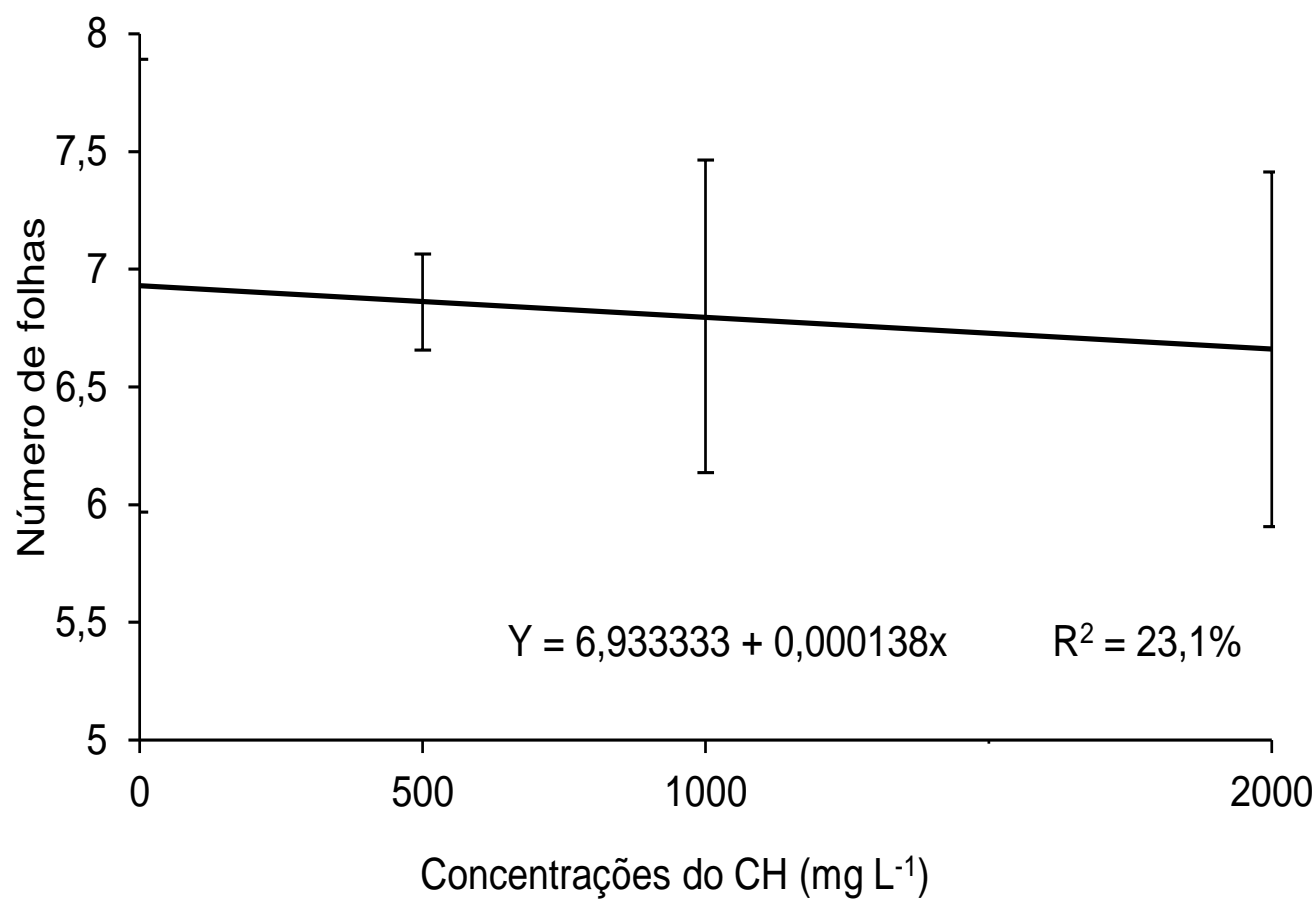

Figura 3 - Número de folhas de mudas de maracujazeiro-azedo submetidas ao tratamento com complexo hidrossolúvel $(\mathrm{CH})$. Médias dos tratamentos com $\mathrm{CH}$ e adubação química. Leaf number of yellow passion fruit seedlings fertilized with water-soluble complex $(\mathrm{CH})$. Averages of treatments with the $\mathrm{CH}$ and the chemistry fertilizer. $(F=0,4300)$. 


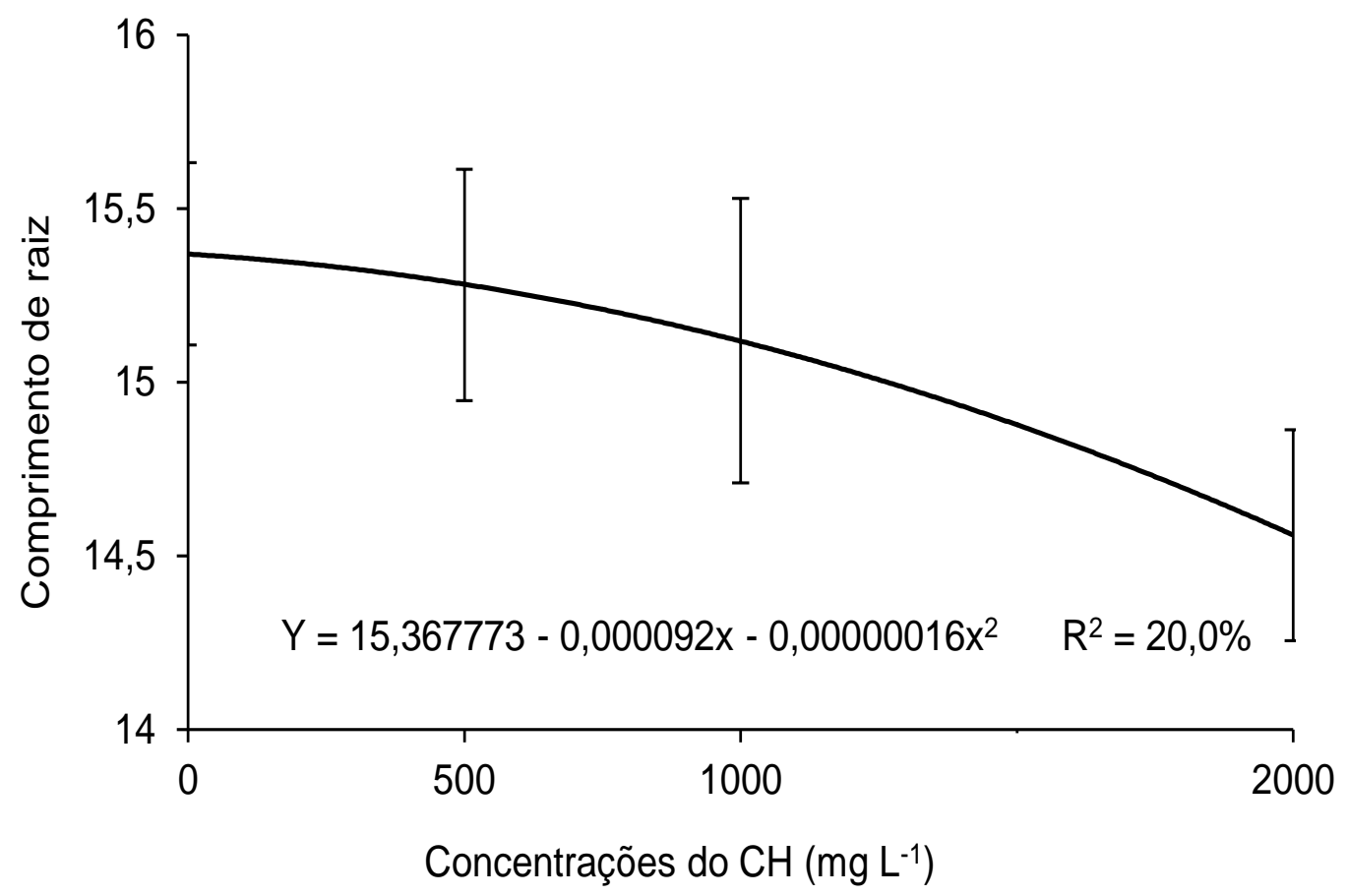

Figura 4 - Comprimento de raiz de mudas de maracujazeiro-azedo submetidas ao tratamento com complexo hidrossolúvel $(\mathrm{CH})$. Médias dos tratamentos com $\mathrm{CH}$ e adubação química. Root lenght of yellow passion fruit seedlings fertilized with water-soluble complex $(\mathrm{CH})$. Averages of treatments with the $\mathrm{CH}$ and the chemistry fertilizer. ( $F=0,3017)$.

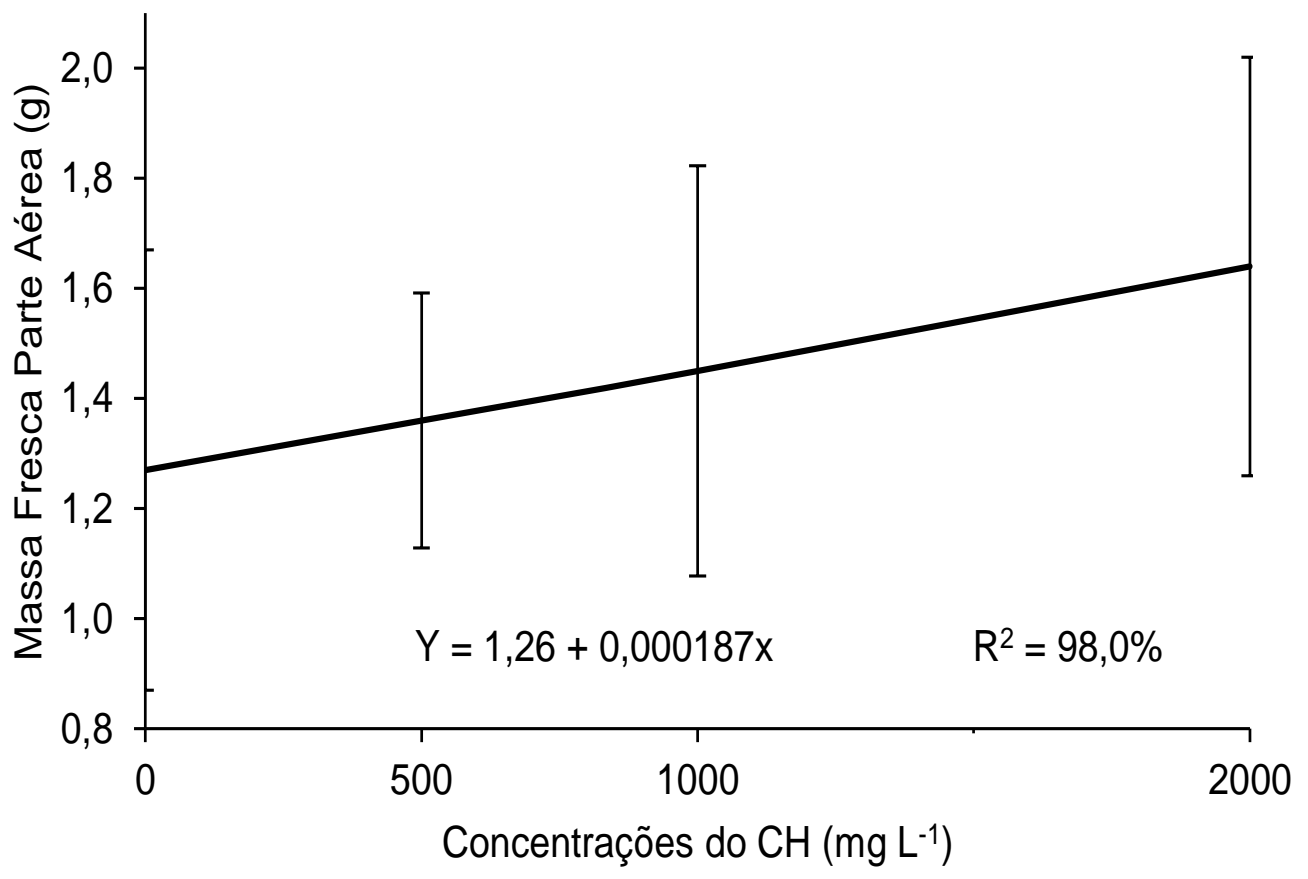

Figura 5 - Massa da matéria fresca da parte aérea de mudas de maracujazeiro submetidas ao tratamento com complexo hidrossolúvel $(\mathrm{CH})$. Médias dos tratamentos com $\mathrm{CH}$ e adubação química. Fresh matter of aerial parts of yellow passion fruit seedlings fertilized with water-soluble complex (CH). Averages of treatments with the $\mathrm{CH}$ and the chemistry fertilizer. $(\mathrm{F}=0,0002)$ 
Para a massa da matéria seca da parte aérea, a regressão demonstrou resultado similar (Figura 6) ao estimado para massa da matéria fresca da parte aérea, como esperado, porém apresentando maior ângulo de inclinação da reta representativa do incremento linear da massa da matéria seca. Essa maior inclinação observada pode estar relacionada com a ausência de água na matéria analisada e ao fato de que, com o aumento das concentrações de aplicação do $\mathrm{CH}$, aumentou-se a assimilação dos nutrientes, tornando a matéria vegetal mais encorpada. $\mathrm{O}$ valor estimado para a maior dosagem testada foi de $0,32 \mathrm{~g}$.

Almeida et al. (2012) também obtiveram aumento em massa da matéria fresca e seca em mudas de maracujazeiro-azedo fertilizadas com esgoto doméstico tratado e ressaltaram que o bom desempenho das mudas estava relacionado ao aproveitamento dos nutrientes presentes no efluente, principalmente N. Já Silva et al. (2010) avaliaram o efeito de substratos e tamanho de recipientes na produção e na qualidade das mudas de maracujazeiro-azedo e obtiveram melhores resultados em altura e massa da matéria seca total da parte aérea quando utilizaram esterco bovino na composição do substrato e, conforme os autores, o suprimento de nutrientes feito por este material foi um dos fatores que influenciaram positivamente.

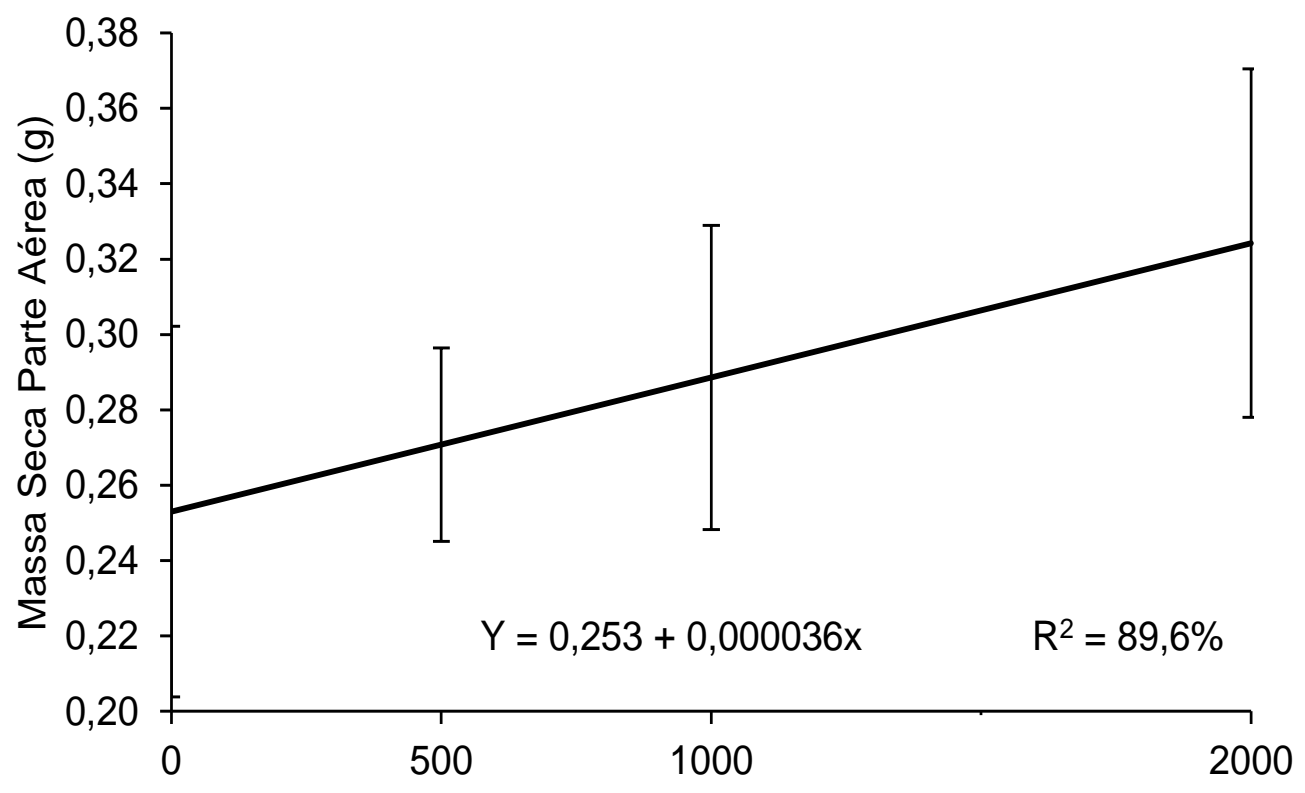

Concentrações do $\mathrm{CH}\left(\mathrm{mg} \mathrm{L}^{-1}\right)$

Figura 6 - Massa da matéria seca da parte aérea de mudas de maracujazeiro submetidas ao tratamento com complexo hidrossolúvel $(\mathrm{CH})$. Médias dos tratamentos com $\mathrm{CH}$ e adubação química. Dry matter of aerial parts of yellow passion fruit seedlings fertilized with water-soluble complex (CH). Averages of treatments with the $\mathrm{CH}$ and the chemistry fertilizer. ( $\mathrm{F}=0,0013)$

Já para a característica massa da matéria fresca de raízes, a regressão demonstrou tendência quadrática da curva (Figura 7), em que o valor máximo estimado foi de $1,67 \mathrm{~g}$ na dosagem de $1.207,1 \mathrm{mg} \mathrm{L}^{-1}$ do $\mathrm{CH}$.

A massa da matéria seca de raízes aumentou de forma linear conforme se aumentavam as concentrações do $\mathrm{CH}$, obtendo-se na maior dosagem $0,16 \mathrm{~g}$ de massa seca de raízes (Figura 8).

A AQC promoveu resultados superiores em todos os parâmetros avaliados, com exceção do comprimento da maior raiz, que não diferiu estatisticamente das mudas tratadas com $\mathrm{CH}$ (Tabela 1).

Entre os elementos mais exigidos pelo maracujazeiro-azedo, citam-se o N, K e Ca (Haag et al., 1973, Lima, 2002, Serrano et al., 2006, Almeida et al., 2012) e ambos foram fornecidos pela adubação química em quantidade recomendada, ou seja, $\mathrm{K}$ e Ca pela adubação básica do substrato e $\mathrm{N}$ em cobertura, o que muito provavelmente explica 0 maior crescimento e desenvolvimento obtidos pelas mudas que receberam a adubação química. É sabido que o $\mathrm{N}$ é importante constituinte de muitos componentes da célula vegetal e promove o crescimento vegetal, que o $\mathrm{K}$ está envolvido no equilíbrio osmótico e iônico, e na regulação estomática, e é ativador de muitas enzimas, e o Ca é componente da parede celular, cofator enzimático e participante do processo de divisão celular (Raven et al., 2001, Lima, 2002, Taiz \& Zeiger, 2009, Tofanelli \& Silva, 2011, Almeida, 2012), sendo consequentemente imprescindíveis para o crescimento e o desenvolvi- 
mento das planas.

O fato de não ter sido observado efeito da adubação química no comprimento da maior raiz talvez esteja relacionado com o uso dos tubetes, cujo comprimento é limitado, e aos 80 DAS, quando se realizaram as avaliações, a maior raiz da maioria das mudas já tivesse atingido o comprimento máximo do recipiente, tendo seu crescimento limitado pelo tamanho do tubete.

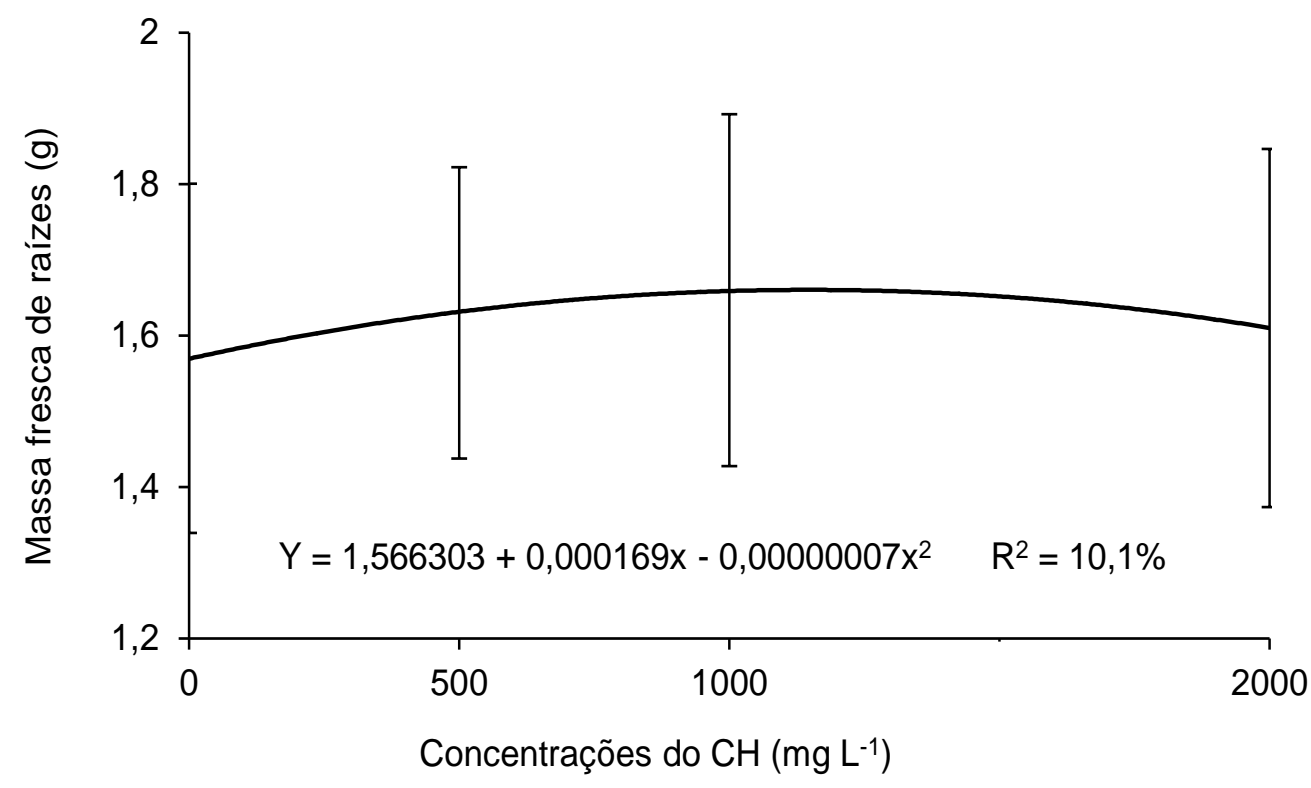

Figura 7 - Massa da matéria fresca de raízes de mudas de maracujazeiro submetidas ao tratamento com complexo hidrossolúvel $(\mathrm{CH})$. Médias dos tratamentos com $\mathrm{CH}$ e adubação química. Fresh matter of roots of yellow passion fruit seedlings fertilized with water-soluble complex $(\mathrm{CH})$. Averages of treatments with the $\mathrm{CH}$ and the chemistry fertilizer. $(F=0,3605)$.

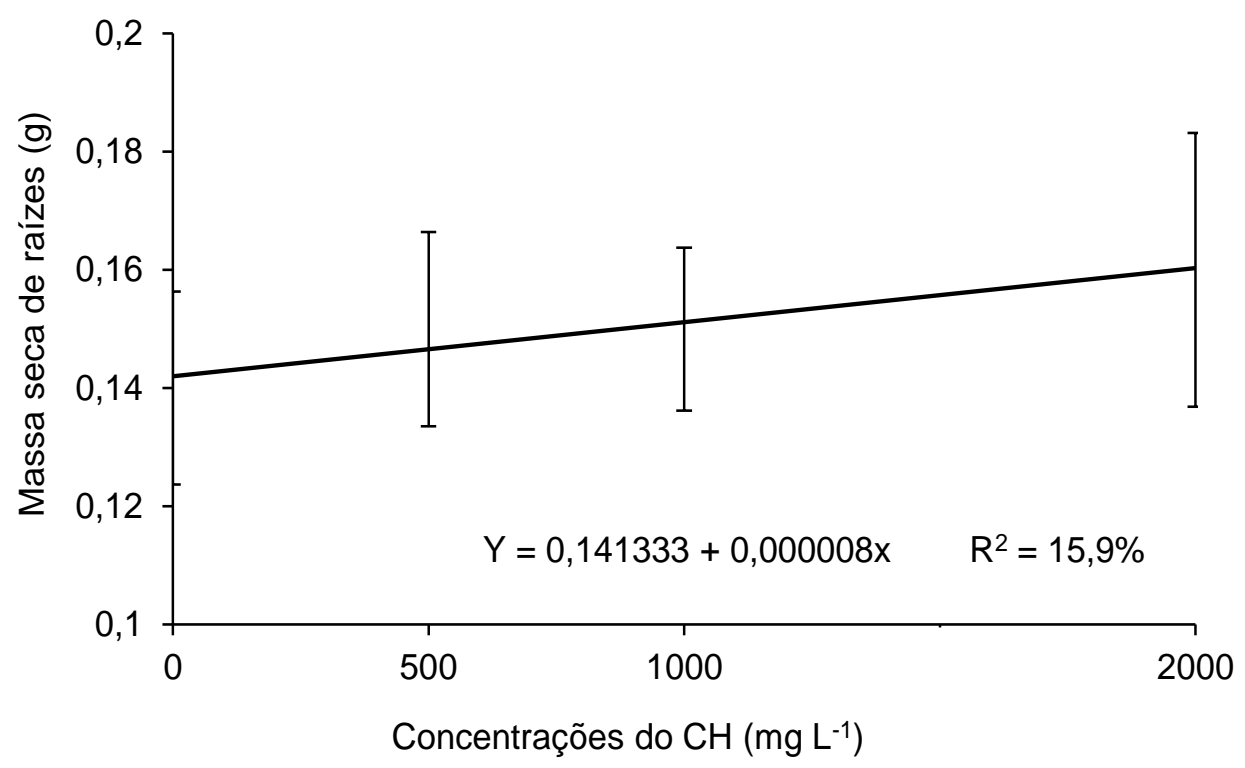

Figura 8 - Massa da matéria seca de raízes de mudas de maracujazeiro submetidas ao tratamento com complexo hidrossolúvel $(\mathrm{CH})$. Médias dos tratamentos com $\mathrm{CH}$ e adubação química. Dry matter of roots of yellow passion fruit seedlings fertilized with water-soluble complex $(\mathrm{CH})$. Averages of treatments with the $\mathrm{CH}$ and the chemistry fertilizer. $(F=0,8431)$. 


\section{Conclusões}

1 - A adubação química convencional (AQC) promove maior crescimento das mudas de maracujazeiro-azedo quando comparado ao complexo hidrossolúvel $(\mathrm{CH})$, com exceção do comprimento da maior raiz.

2 - $\mathrm{O} \mathrm{CH}$ aumenta a qualidade das mudas de maracujazeiro-azedo, especificamente, melhorando sua altura e sua massa da matéria vegetal da parte aérea.

3 - A aplicação do $\mathrm{CH}$ na concentração de $2.000 \mathrm{mg} \mathrm{L}^{-1}$ melhora a qualidade das mudas de maracujazeiro-azedo.

4 - $\mathrm{O} \mathrm{CH}$ pode ser usado como complemento da adubação química na formação de mudas de maracujazeiro-azedo, porém não deve ser a única fonte de adubação.

\section{Referências}

Albuquerque RP de F, Pereira WE, Marques LF, Araujo RC, Lopes EB (2010) Crescimento e composição mineral de mudas de maracujazeiro azedo fertilizados com boro e potássio. Revista Engenharia Ambiental: Pesquisa e Tecnologia 7(2):84-96.

Almeida EV, Natale W, Prado RM, Barbosa JC (2006) Adubação nitrogenada e potássica no desenvolvimento de mudas de maracujazeiro. Ciência Rural 36(4):1138-1142.

Almeida JPN de, Costa LR da, Sampaio PRF, Azevedo J de, Dias N da S (2012) Utilização de esgoto doméstico tratado na produção de mudas de maracujazeiro azedo. Revista Verde 7(4):69-75.

Almeida M de O, Cruz M do CM, Castro GDM de, Fagundes MCP (2014) Crescimento e absorção de nutrientes por mudas de maracujazeiro-amarelo em substratos orgânico e comercial e adubação nitrogenada. Revista Brasileira de Ciências Agrárias 9(2):180-185.

Almeida RF de (2012) Nutrição de maracujazeiro. Revista Verde 7(3):12-17.

Assis RU de (2002) Agroecologia no Brasil: análise do processo de difusão e perspectivas (Tese de doutorado em Economia Aplicada)

Bernardi MR, Junior MS, Daniel O, Vitorino ACT (2012) Crescimento de mudas de Corymbia citriodora em função do uso de hidrogel e adubação. Cerne 18(1):67-74.

Costa E, Santos LCR dos, Carvalho C de, Leal PAM, Gomes V do A (2011) Volumes de substratos comerciais, solo e composto orgânico afetando a formação de mudas de maracujazeiro-amarelo em diferentes. Revista Ceres 58(2):216-222.
Cruz M do CM da, Ramos JD, Oliveira DL de, Marques VB, Hafle OM (2008) Utilização de água residuária de suinocultura na produção de mudas de maracujazeiro-azedo cv Redondo Azedo. Revista Brasileira de Fruticultura 30(4):1107-1112.

Gurgel RL da S, Souza HA de, Teixeira GA, Mendonça V, Ferreira EA (2007) Adubação fosfatada e composto orgânico na produção de mudas de maracujazeiro-amarelo. Revista Brasileira de Ciências Agrárias 2(4):262-267.

Haag HP, Oliveira GD de, Borducchi AS, Sarruge JR (1973) Absorção de nutrientes por duas variedades de maracujá. Anais... 30:267-279.

Hartmann HT, Kester DE, Davies Junior FT, Geneve $\mathrm{RL}$ (2010) Plant propagation: principles and practices. Prentice-Hall. 928p.

IBGE - Instituto Brasileiro de Geografia e Estatística (2013) Sistema IBGE de recuperação automática Sidra. Disponível em: <http://www.sidra.ibge.gov.br> (Acesso em 15 abr. 2014).

Inocêncio MF, Novelino JO, Paim LR, Gutierrez RS (2009) Efeito da aplicação de basalto triturado nas características químicas de amostras de solo do estado de Mato Grosso do Sul. Revista Caatinga 22(4):145-151.

Kirkby EA, Römheld V (2007) Micronutrientes na fisiologia de plantas: funções, absorção e mobilidade. Encarte Técnico. 24p. Disponível em: <http://www.ipni.net/publication/ia-brasil.nsf> (Acesso em 05 abr 2013).

Leite GA, Medeiros LF, Pereira FS, Linhares PCF, Mendonça V, Maracaja PB, Fernandes AA (2011) Desenvolvimento inicial de mudas de maracujazeiro azedo sob diferentes proporções de jitirana (Merremia aegyptia L.) incorporadas ao substrato. Agropecuária Científica no Semi-Árido (7):32-36.

Lima A de A (2002). Maracujá: produção - aspectos técnicos. Frutas do Brasil 15. Embrapa Informação Tecnológica. 104p.

Mendonça V, Ferreira EA, Paula YCM, Batista TMV, Ramos JD (2007) Crescimento de mudas de maracujazeiro-azedo influenciado por doses de nitrogênio e de superfosfato simples. Revista Caatinga 20:137-143.

Mesquita F de O, Rebequi AM, Cavalcante LF, Souto AG de $L$ (2012) Crescimento absoluto e relativo de mudas de maracujazeiro sob biofertilizante e águas salinas. Revista de Ciências Agrárias 35(1):222-239.

Müller-Stecz Indústria e Comércio (2011) Indústria. Piraquara. Disponível em: < http://www.steczcom.blogspot.com.br>. (Acesso em: 09 set. 2015). 
Natale W, Prado RM, Almeida E, Barbosa JC (2006) Adubação nitrogenada e potássica no estado nutricional de mudas de maracujazeiro-azedo. Acta Scientiarum. Agronomy 28(2):187-192.

Pereira HS, Vitti GC (2004) Efeito do uso do xisto em características químicas do solo e nutrição do tomateiro. Horticultura Brasileira 22(2):317-322.

Pereira WE, Lima SF, Paula LB, Alva-Rez VVH (2000) Crescimento e composição mineral de mudas de maracujazeiro em função de doses de osmocote em dois tipos de substratos. Revista Ceres 47(271):311-324.

Profeta GA, Braga MJ (2011) Poder de mercado na indústria brasileira de fertilizantes npk (04-14-08), no período de 1993-2006. Revista de Economia e Sociologia Rural 49(4):837-856.

Raven PH, Evert RF, Eichhorn SE (2001) Biologia Vegetal. Guanabara-Koogan. 906p.

Rodolfo Junior F, Cavalcante LF, Buriti E de S (2008) Crescimento e produção do maracujazeiro-amarelo em solo com biofertilizantes e adubação mineral com npk. Caatinga 21(5):134-145.

Sá JR de, Oliveira AES de, Medeiro JF de, Nogueira NW, Silva CB e (2010) Interação da adubação organo-mineral nos atributos químicos do solo na cultura do melão em Mossoró - RN - Brazil. Revista Verde 5(3):89-100.

Sampaio ES de (1998) Fisiologia vegetal: teoria e experimentos. Editora UEPG. 190p.

Serrano LAL, Silva CMM da, Ogliari J, Carvalho AJC de, Marinho CS, Detmann E (2006) Utilização de substrato composto por resíduos da agroindústria canavieira para produção de mudas de maracujazeiro-azedo. Revista Brasileira de Fruticultura 28(3):487-491.
Silva EA da, Maruyama WI, Mendonça V, Francisco MGS, Bardiviesso DM, Tosta M da S (2010) Composição de substratos e tamanho de recipientes na produção e qualidade das mudas de maracujazeiro 'azedo'. Ciência e Agrotecnologia 34(3):588-595.

Silva JR da (2004) Maracujá: Produção, pós-colheita e mercado. Instituto Frutal. 77p.

Sousa GG de, Novelino JO, Scalon SQ de P, Marchetti ME (2011) Crescimento de mudas de maracujazeiro em função de adubação à base de boro e material de cupinzeiro. Pesquisa Agropecuária Tropical 41(2): 170-178.

Taiz L, Zeiger E (2009) Fisiologia vegetal. Artmed Editora. 819p.

Tofanelli MBD, Silva TO da (2011) Manejo ecológico e conservação dos solos e da água no estado de Sergipe. Editora UFS. 358p.

Valadão A da C, Finkler CN, Chebinski C, Pinto CFB, Tardin JM, Monnerat PF, Moreira S dos S, Faria LG (2006) Agroecologia: a organização camponesa reconstruindo o sustento da vida e a transformação da sociedade. Lance Livre Design, Produções e Editora. 149p.

Wagner Júnior A, Santos CEM dos, Silva JO da C, Pimentel LD, Bruckner CH, Mazaro SM (2011) Densidade de sementes de três espécies de maracujazeiro na emergência e desenvolvimento inicial das plântulas. Revista Brasileira de Agrociência 17(3-4):359-364. 